\title{
Avaliação da atuação da rede comunitária de saúde mental em um município paulista de médio porte
}

\author{
Evaluation of the performance of the community mental health \\ network in a medium-sized municipality from the state of São Paulo
}

Carlos Alberto dos Santos Treichel' ${ }^{\mathbf{1}}$ Rosana Teresa Onocko Campos $\mathbf{1}$

DOI: $10.1590 / 0103-1104202213208$

\begin{abstract}
RESUMO Tendo como norteadores as recentes mudanças na condução das políticas de saúde mental no País e as disparidades em saúde vivenciadas por pessoas que convivem com transtornos mentais, este estudo buscou avaliar a atuação de uma rede de saúde mental comunitária a partir de três componentes principais: 1) a caracterização do perfil dos usuários; 2) a identificação das ofertas terapêuticas; e 3) a identificação dos cuidados clínicos recebidos pelos usuários em cada um dos serviços estudados, a saber: Centro de Atenção Psicossocial-II; Centro de Atenção Psicossocial-AD; ambulatório; e 13 serviços de Atenção Básica. Trata-se de um estudo transversal baseado em documentos, realizado através da análise de 4582 prontuários. Foram evidenciadas diferenças importantes entre os serviços quanto ao seu funcionamento e diálogo com outros pontos da rede de atenção. As ofertas terapêuticas foram caracterizadas, principalmente, pela oferta de consultas médicas e consultas individuais com profissionais da equipe multidisciplinar. Houve baixa utilização dos recursos comunitários e das oficinas terapêuticas. Foi observada, ainda, baixa oferta de cuidados clínicos aos usuários dos serviços especializados, em contraponto com a Atenção Básica, que, por sua vez, mostrou-se como um serviço potente e necessário para o cuidado das pessoas com transtornos mentais.
\end{abstract}

PALAVRAS-CHAVE Saúde mental. Serviços comunitários de saúde mental. Atenção Primária à Saúde. Pesquisa sobre serviços de saúde. Avaliação em saúde.

\begin{abstract}
Having as guiding elements the recent changes in the conduction of mental health policies in the Country and the health disparities experienced by people living with mental disorders, this study sought to evaluate the performance of a community mental health network based on three main components: 1) the characterization of the users' profile; 2) identification of therapeutic offers; and 3) identification of the clinical care received by users in each of the services studied, namely: Psychosocial Care Center-II; Psychosocial Care Center-AD; outpatient clinic; and 13 Primary Care services. This is a cross-sectional study based on documents, carried out through the analysis of 4582 medical records. Important differences were evidenced between the services regarding their functioning and dialogue with other points of the care network. The therapeutic offers were mainly characterized by the offer of medical consultations and individual consultations with professionals from the multidisciplinary team. There was low use of community resources and therapeutic workshops. It was observed, as well, a low offer of clinical care to users of specialized services, in contrast to Primary Care, which, in turn, proved to be a powerful and necessary service for the care of people with mental disorders.
\end{abstract}

1 Universidade Estadual de Campinas (Unicamp) Campinas (SP), Brasil. carlos-treichel@hotmail.com
KEYWORDS Mental health. Community mental health services. Primary Health Care. Health services research. Health evaluation. 


\section{Introdução}

Estima-se que, aproximadamente, 6,9 milhões de brasileiros apresentem transtornos mentais graves e persistentes, demandando, assim, cuidados intensivos e contínuos em saúde mental' ${ }^{1}$. Além disso, outros 30,2 milhões convivem com transtornos leves ou moderados que requerem tratamento ocasional em serviços especializados ${ }^{2}$. Nesse contexto, os serviços comunitários têm sido importantes protagonistas do cuidado. Atualmente, além dos serviços de Atenção Básica (AB), o Brasil conta com mais de 2.300 Centros de Atenção Psicossocial (Caps) ${ }^{3}$.

Os Caps são regulamentados pela Portaria $\mathrm{n}^{\circ} 3.088$, de 23 de dezembro de $2011^{4}$, e foram instituídos enquanto serviços estratégicos e prioritários do modelo de atenção implantado no País por meio da Reforma Psiquiátrica vivenciada a partir da década de 1980 . Tendo um papel de coordenação na rede de saúde mental, esses serviços seriam responsáveis pela admissão de pessoas com transtornos mentais, oferecendo cuidados clínicos e de reabilitação psicossocial ${ }^{3}$. Contudo, a partir das disputas políticas que permearam o País nos anos recentes, houve uma série de retrocessos na condução das políticas de saúde mental ${ }^{5}$.

A partir da Portaria ${ }^{0} 3.588$, de 21 de dezembro de $2017^{6}$, entre as principais alterações na organização dos serviços, destaca-se a ratificação dos ambulatórios especializados e das Comunidades Terapêuticas (CTs) como parte integrante da Rede de Atenção Psicossocial (Raps). Se, por um lado, essas incorporações aparentam revidar a lacuna de cuidados em saúde mental vivenciada no País, por outro lado, apontam para o flerte incoerente com o modelo biomédico, representado pela hierarquização e desarticulação dos ambulatórios ${ }^{5}$, e com o modelo de tratamento moral instigado pelas CTs, que, além de promoverem o afastamento do indivíduo da sociedade, são, em grande parte, pautadas pela religião e não possuem equipes técnicas ${ }^{7}$.

Trata-se, portanto, de um movimento em oposição aos objetivos fundamentais do Plano Global de Saúde Mental da Organização Mundial da Saúde (OMS) ${ }^{8}$ e do relatório da Comissão Lancet sobre saúde mental global e desenvolvimento sustentável9 ${ }^{9}$ Em um momento em que existe um esforço global com vistas a fortalecer a $\mathrm{AB} e$ a integração dos serviços especializados para o atendimento em saúde mental, essas mudanças trazem uma complexidade extra ao estudo da Raps no Brasil.

Atualmente, estima-se que pessoas convivendo com transtornos mentais tenham uma expectativa de vida reduzida entre 12 e 20 anos quando comparadas com a população geral10. Os fatores que explicam essa realidade incluem os efeitos adversos da medicação, bem como os comportamentos não saudáveis no estilo de vida, que aumentam a probabilidade de desenvolverem obesidade e hipercolesterolemia, que, por sua vez, aumentam o risco de doenças crônicas como hipertensão, diabetes mellitus ou mesmo síndrome metabólica"1. Dessa forma, a fim de reduzir as disparidades em saúde vivenciadas por essa população, é necessário cada vez mais que os serviços providenciem atividades oportunas e apropriadas de prevenção, promoção e monitoramento da saúde.

O objetivo deste estudo foi avaliar a atuação de uma rede de saúde mental comunitária em um município paulista de médio porte através da: 1) caracterização do perfil dos usuários; 2) identificação das ofertas terapêuticas; e 3) identificação dos cuidados clínicos recebidos pelos usuários em cada um dos serviços estudados, a saber: Centro de Atenção PsicossocialII (Caps-II); Centro de Atenção Psicossocial Álcool e outras Drogas (Caps-AD); ambulatório; e 13 serviços de $\mathrm{AB}$ que operam no modelo de Estratégia Saúde da Família (ESF).

\section{Material e métodos}

\section{Design de estudo}

Trata-se de um estudo transversal baseado em documentos, realizado através da análise 
de prontuários de usuários dos serviços ambulatoriais especializados em saúde mental e serviços de $\mathrm{AB}$ que operam no modelo de ESF no município estudado.

\section{População e seleção de participantes}

A partir de um levantamento conduzido previamente, foi possível identificar que, durante o período da pesquisa, 413 usuários estavam frequentando o Caps-II, 403 usuários estavam frequentando o Caps-AD, 1.142 pacientes estavam recebendo consultas de saúde mental no Ambulatório Central de Especialidades e 3.022 usuários estavam recebendo consultas de saúde mental nos 13 serviços de ESF.

O critério de inclusão neste estudo foi ter pelo menos um registro de atendimento em saúde mental entre os meses de maio de 2018 e maio de 2019. Não ser maior de idade foi considerado um critério de exclusão. Dessa forma, foram incluídos no estudo 4.582 usuários da rede de serviços de saúde mental do município em nível ambulatorial e comunitário.

\section{Procedimentos para coleta de dados}

Os procedimentos para coleta de dados foram realizados nos serviços estudados entre maio e julho de 2019 por 16 coletadores previamente treinados. Esses coletadores foram selecionados entre estudantes profissionais dos cursos de psicologia e medicina e foram supervisionados por uma enfermeira e uma assistente social. Quando atendidos os critérios de inclusão, os coletadores preencheram com base nas informações disponíveis nos prontuários um formulário elaborado especificamente para os fins desta pesquisa

\section{Variáveis de estudo}

A fim de responder aos objetivos do estudo, com base nas informações dos prontuários, foram selecionadas para estudo as seguintes variáveis: sexo (feminino, masculino); idade (18 a 30 anos, 31 a 45 anos, 46 a 60 anos, 61 anos ou mais); escolaridade (0 a 4 anos, 5 a 8 anos, 9 anos ou mais, sem registro); trabalho remunerado (sim, não, sem registro); tempo em atendimento ( 1 a 6 meses, 7 a 12 meses, 13 a 36 meses, 37 a 60 meses, 61 meses ou mais); origem do encaminhamento (demanda espontânea, AB, serviço hospitalar ou emergencial, outro serviço especializado, serviços privados); diagnóstico (transtornos neuróticos, transtornos afetivos - unipolares, transtornos afetivos - bipolares, transtornos psicóticos, uso abusivo de Substâncias Psicoativas (SPA), retardo mental e transtornos orgânicos, sem diagnóstico); número de atendimentos de saúde mental ( 1 a 3 atendimentos, 4 a 6 atendimentos, 6 a 12 atendimentos, 13 atendimentos ou mais); ofertas terapêuticas (consulta médica de saúde mental, atendimento individual com Profissional da Equipe Multidisciplinar (PEM), atendimento coletivo, oficina terapêutica/geração de renda, atividade comunitária, atendimento domiciliar, atividade física); cuidados clínicos recebidos (aferição do peso, aferição dos sinais vitais, anamnese e exame físico, exames laboratoriais, tratamentos clínicos/encaminhamentos); hipertensão (sim, não, sem registro); diabetes (sim, não, sem registro); tabagismo (sim, não, sem registro); etilismo (sim, não, sem registro); uso de outras SPA (sim, não, sem registro).

As variáveis relativas às ofertas terapêuticas e aos cuidados clínicos recebidos foram avaliadas com base nos registros do ano que antecedeu a coleta de dados, ou seja, foi avaliado o período de maio de 2018 a maio de 2019.

\section{Análise estatística}

As análises estatísticas foram realizadas com uso do software Stata 15 (Stata Corporation, College Station, Texas, EUA). Foi utilizada análise estatística descritiva com estimativas das frequências absoluta e relativa para cada uma das variáveis estudadas. Como a falta de determinadas informações era considerada relevante para discussão do estudo, os dados faltantes (missings) foram computados e relatados. 


\section{Procedimentos éticos}

Este estudo foi submetido e aprovado pelo Comitê de Ética em pesquisa da Faculdade de Ciências Médicas da Universidade Estadual de Campinas sob parecer de $n^{0}$ 3.793.771. Sua realização se deu seguindo as normas e diretrizes brasileiras de regulamentação para pesquisas envolvendo seres humanos (Resolução n ${ }^{0} 466 / 2012$ do Conselho Nacional de Saúde) ${ }^{\mathbf{1 2}}$, além das disposições da Declaração de Helsinque. O anonimato dos participantes foi assegurado.

\section{Resultados}

Ao total, 4.582 usuários da rede de serviços de saúde mental, em nível ambulatorial e comunitário, foram incluídos no estudo. Destes, 9,0\% $(n=413)$ estavam vinculados ao Caps-II, 7,8\% $(\mathrm{n}=359)$ ao Caps-AD, 22,5\% (n=1.032) ao ambulatório e $60,6 \%(n=2.778)$ à $A B$. A maior parte dos usuários era formada por mulheres $(68,8 \%$, n=3.152), e a média de idade correspondeu a 49,4 $(\mathrm{DP}=16,4)$. A caracterização dos usuários incluídos no estudo, de acordo com o serviço ao qual estão vinculados, pode ser observada na tabela 1.

Tabela 1. Caracterização dos usuários incluídos no estudo de acordo com o ponto de atenção a que estão vinculados $(n=4582)$

\begin{tabular}{|c|c|c|c|c|c|c|c|c|}
\hline & \multicolumn{2}{|c|}{ Caps-II } & \multicolumn{2}{|c|}{ Caps-AD } & \multicolumn{2}{|c|}{ Ambulatório } & \multicolumn{2}{|c|}{ Atenção Básica } \\
\hline & $\mathrm{n}$ & $\%$ & $\mathrm{n}$ & $\%$ & $\mathrm{n}$ & $\%$ & $\mathrm{n}$ & $\%$ \\
\hline \multicolumn{9}{|l|}{ Sexo } \\
\hline Feminino & 227 & 55,0 & 80 & 22,3 & 741 & 71,8 & 2104 & 75,7 \\
\hline Masculino & 186 & 45,0 & 279 & 77,7 & 291 & 28,2 & 674 & 24,3 \\
\hline \multicolumn{9}{|l|}{ Idade } \\
\hline 18 a 30 anos & 54 & 13,1 & 101 & 28,1 & 127 & 12,3 & 361 & 12,9 \\
\hline 31 a 45 anos & 127 & 30,8 & 138 & 38,5 & 306 & 29,7 & 724 & 26,1 \\
\hline 46 a 60 anos & 162 & 39,2 & 102 & 28,4 & 325 & 31,5 & 852 & 30,7 \\
\hline 61 anos ou mais & 70 & 16,9 & 18 & 5,0 & 274 & 26,5 & 841 & 30,3 \\
\hline \multicolumn{9}{|l|}{ Escolaridade } \\
\hline 0 a 4 anos & 168 & 40,7 & 29 & 8,1 & 299 & 29,0 & 312 & 11,2 \\
\hline 5 a 8 anos & 43 & 10,4 & 16 & 4,5 & 163 & 15,8 & 227 & 8,2 \\
\hline 9 anos ou mais & 104 & 25,2 & 17 & 4,7 & 259 & 25,1 & 310 & 11,2 \\
\hline Sem Registro & 98 & 23,7 & 297 & 82,7 & 311 & 30,1 & 1929 & 69,4 \\
\hline \multicolumn{9}{|c|}{ Trabalho Remunerado } \\
\hline Sim & 47 & 11,4 & 98 & 27,3 & 10 & 1,0 & 99 & 3,6 \\
\hline Não & 185 & 44,8 & 73 & 20,3 & 5 & 0,5 & 71 & 2,5 \\
\hline Sem Registro & 181 & 43,8 & 188 & 52,4 & 1017 & 98,5 & 2608 & 93,9 \\
\hline \multicolumn{9}{|c|}{ Tempo em atendimento } \\
\hline 1 a 6 meses & 47 & 11,4 & 134 & 37,3 & 148 & 14,3 & 261 & 9,4 \\
\hline 7 a 12 meses & 30 & 7,2 & 88 & 24,5 & 160 & 15,5 & 270 & 9,7 \\
\hline 13 a 36 meses & 74 & 17,9 & 38 & 10,6 & 252 & 24,4 & 408 & 14,7 \\
\hline 37 a 60 meses & 61 & 14,8 & 29 & 8,1 & 465 & 45,1 & 315 & 11,3 \\
\hline 61 meses ou mais & 201 & 48,7 & 70 & 19,5 & 7 & 0,7 & 1524 & 54,9 \\
\hline
\end{tabular}


Tabela 1. (cont.)

\begin{tabular}{|c|c|c|c|c|c|c|c|c|}
\hline & \multicolumn{2}{|c|}{ Caps-II } & \multicolumn{2}{|c|}{ Caps-AD } & \multicolumn{2}{|c|}{ Ambulatório } & \multicolumn{2}{|c|}{ Atenção Básica } \\
\hline & n & $\%$ & $\mathbf{n}$ & $\%$ & $\mathbf{n}$ & $\%$ & $\mathrm{n}$ & $\%$ \\
\hline \multicolumn{9}{|l|}{ Origem do encaminhamento } \\
\hline Demanda espontânea & 64 & 15,5 & 181 & 50,4 & 740 & 71,7 & 0 & 0 \\
\hline Atenção Básica & 70 & 16,9 & 43 & 12,0 & 267 & 25,9 & 2731 & 98,3 \\
\hline Serviço hospitalar ou emergencial & 47 & 11,4 & 14 & 3,9 & 4 & 0,4 & 9 & 0,3 \\
\hline Outro serviço especializado & 206 & 49,9 & 32 & 8,9 & 11 & 1,0 & 30 & 1,1 \\
\hline Serviços privados & 26 & 6,3 & 89 & 24,8 & 10 & 1,0 & 8 & 0,3 \\
\hline \multicolumn{9}{|l|}{ Diagnóstico* } \\
\hline Transtornos neuróticos & 66 & 16,0 & 45 & 12,5 & 573 & 55,5 & 1040 & 37,4 \\
\hline Transtornos afetivos (unipolares) & 71 & 17,2 & 25 & 6,9 & 228 & 22,1 & 640 & 23,0 \\
\hline Transtornos afetivos (bipolares) & 73 & 17,7 & 8 & 2,2 & 41 & 4,0 & 20 & 0,7 \\
\hline Transtornos psicóticos & 172 & 41,6 & 12 & 3,3 & 58 & 5,6 & 81 & 2,9 \\
\hline Uso abusivo de SPA & 17 & 4,1 & 131 & 36,5 & 41 & 4,0 & 69 & 2,5 \\
\hline Retardo mental e transtornos orgânicos & 54 & 13,1 & 11 & 3,1 & 64 & 6,2 & 68 & 2,4 \\
\hline Sem diagnóstico & 49 & 11,9 & 224 & 62,4 & 280 & 27,1 & 1110 & 39,9 \\
\hline \multicolumn{9}{|l|}{ Número de atendimentos de SM } \\
\hline 1 a 3 atendimentos & 99 & 24,0 & 192 & 53,5 & 680 & 65,9 & 1912 & 68,8 \\
\hline 4 a 6 atendimentos & 254 & 61,5 & 66 & 18,4 & 274 & 26,5 & 633 & 22,8 \\
\hline 6 a 12 atendimentos & 46 & 11,1 & 53 & 14,7 & 69 & 6,7 & 217 & 7,8 \\
\hline 13 atendimentos ou mais & 14 & 3,4 & 48 & 13,4 & 9 & 0,9 & 16 & 0,6 \\
\hline
\end{tabular}

Fonte: elaboração própria.

*Todos os diagnósticos atribuídos foram considerados. Alguns usuários possuíam até 3 diagnósticos. SM= Saúde Mental.

Houve predomínio do sexo feminino em todos os serviços, exceto no Caps-AD, cuja proporção de usuários do sexo masculino correspondeu a 77,7\% $(n=279)$. Com relação ao Caps-AD, destaca-se, ainda, uma maior proporção de usuários mais jovens, especialmente quando comparados aos usuários da $\mathrm{AB}$, onde a proporção de usuários entre 46 e 60 anos e com 61 anos ou mais correspondeu a 33,7\% $(n=852)$ e $33,3 \%(n=841)$, respectivamente.

Uma vez que as informações de mais da metade dos usuários não estavam disponíveis no Caps- $A D$ e na $A B$ no que se refere à escolaridade, não foi possível identificar o nível de escolaridade desses usuários. O mesmo ocorreu para a variável trabalho remunerado, na qual se incluem, também, os usuários do ambulatório. No entanto, os dados disponíveis sinalizam para baixa escolaridade dos usuários do Caps-II, onde 40,7\% (n=168) deles possuíam entre 0 e 4 anos de estudo.

A maior parte dos usuários vinculados ao Caps-II e à $\mathrm{AB}$ era atendida no serviço há mais de 5 anos: $48,7 \%(n=201)$ e $54,9 \%(n=1524)$, respectivamente. Usuários do ambulatório estavam no serviço majoritariamente entre 3 e 5 anos (45,1\%, $\mathrm{n}=465)$. Por outro lado, mais da metade dos usuários do Caps-AD frequentava o serviço por até um ano. Quanto à origem do encaminhamento, destacou-se no Caps-AD e no ambulatório a demanda espontânea, correspondendo a 50,4 $(\mathrm{n}=181)$ e $71,7 \%(\mathrm{n}=740)$ dos usuários, respectivamente. Neste segundo, destacou-se, ainda, a proporção de usuários encaminhados pela $\mathrm{AB}(25,9 \%$, $\mathrm{n}=267$ ), enquanto no Caps-AD houve, ainda, 
uma proporção relevante de encaminhamentos realizados por serviços privados $(24,8 \%, \mathrm{n}=89)$. Entre os usuários do Caps-II, destacaram-se os encaminhamentos realizados por outros serviços especializados, correspondentes a 49,9\% $(\mathrm{n}=206)$ dos casos. Já na AB, a ampla maioria dos usuários já era vinculada ao serviço inicialmente $(98,3 \%, \mathrm{n}=2731)$.

No que se refere ao diagnóstico dos usuários, no Caps-II, destaca-se a alta prevalência de transtornos psicóticos (41,6\%, n=172), enquanto no ambulatório e na $\mathrm{AB}$ predominaram os transtornos neuróticos e afetivos unipolares. No Caps-AD, 36,5\% dos usuários possuíam diagnóstico relativo ao uso abusivo de SPA, contudo, destaca-se a alta proporção de usuários sem diagnóstico atribuído
$(62,4 \%, \mathrm{n}=224)$. O mesmo aconteceu para o ambulatório e para a $\mathrm{AB}$, onde $27,1 \%(\mathrm{n}=280)$ e $39,9 \%$ ( $\mathrm{n}=1110$ ) dos usuários não possuíam diagnóstico, respectivamente.

A maior parte dos usuários vinculados ao Caps-AD (53,5\%, $\mathrm{n}=192)$, ao ambulatório $(65,9 \%, \mathrm{n}=680)$ e à $\mathrm{AB}(68,8 \%, \mathrm{n}=1912)$ havia recebido entre 1 e 3 atendimentos no ano que antecedeu a pesquisa. Já no Caps-II, a maior parte dos usuários consultou mais frequentemente, de forma que $61,5 \%(n=254)$ dos usuários receberam entre 4 a 6 atendimentos no ano. Os dados relativos às ofertas terapêuticas, bem como aos cuidados clínicos recebidos pelos usuários em cada ponto de atenção podem ser observados na tabela 2 .

Tabela 2. Ofertas terapêuticas e cuidados clínicos recebidos pelos usuários incluídos no estudo de acordo com o ponto de atenção a que estão vinculados ( $n=4582)$

\begin{tabular}{|c|c|c|c|c|c|c|c|c|}
\hline & \multicolumn{2}{|c|}{ Caps-II } & \multicolumn{2}{|c|}{ Caps-AD } & \multicolumn{2}{|c|}{ Ambulatório } & \multicolumn{2}{|c|}{ Atenção Básica } \\
\hline & n & $\%$ & $\mathbf{n}$ & $\%$ & $\mathbf{n}$ & $\%$ & $\mathbf{n}$ & $\%$ \\
\hline \multicolumn{9}{|l|}{ Ofertas terapêuticas } \\
\hline Consulta médica de saúde mental & 410 & 99,3 & 209 & 58,2 & 1001 & 97,0 & 1947 & 70,1 \\
\hline Atendimento individual (PEM) & 372 & 90,1 & 232 & 89,9 & 293 & 28,4 & 1851 & 66,6 \\
\hline Atendimento coletivo & 37 & 8,96 & 196 & 54,6 & 0 & 0 & 122 & 4,4 \\
\hline Oficina terapêutica/geração de renda & 38 & 9,2 & 24 & 6,6 & 0 & 0 & 15 & 0,5 \\
\hline Atividade comunitária & 11 & 2,7 & 0 & 0 & 0 & 0 & 287 & 10,3 \\
\hline Atendimento domiciliar & 26 & 6,3 & 0 & 0 & 0 & 0 & 233 & 8,4 \\
\hline Atividade física & 6 & 1,4 & 3 & 0,8 & 0 & 0 & 61 & 2,2 \\
\hline \multicolumn{9}{|l|}{ Cuidados clínicos recebidos } \\
\hline Aferição do peso & 64 & 15,5 & 48 & 13,4 & 97 & 9,4 & 2602 & 93,6 \\
\hline Aferição dos sinais vitais & 30 & 7,3 & 141 & 39,3 & 43 & 4,2 & 2574 & 92,7 \\
\hline Anamnese e exame físico & 244 & 59,1 & 222 & 61,8 & 180 & 14,4 & 2630 & 94,7 \\
\hline Exames laboratoriais & 67 & 16,2 & 45 & 12,5 & 124 & 12,2 & 2144 & 77,2 \\
\hline Tratamentos clínicos/encaminhamentos & 72 & 17,4 & 29 & 8,1 & 88 & 8,5 & 1925 & 69,3 \\
\hline
\end{tabular}

Fonte: elaboração própria.

PEM= Profissional da equipe multidisciplinar.

Enquanto no Caps-II houve uma alta proporção de usuários que receberam consulta médica de saúde mental $(99,3 \%, \mathrm{n}=410)$ e atendimento individual com PEM (90,1\%, $\mathrm{n}=372$ ), no Caps AD, além dos atendimentos individuais com PEM, houve destaque para os atendimentos coletivos $(89,9 \%, \mathrm{n}=232)$. Por outro lado, apenas $58,2 \%(n=209)$ dos 
usuários do Caps-AD haviam recebido consultas médicas no último ano. No ambulatório, os atendimentos se configuraram exclusivamente como consultas médicas ( $97 \%, \mathrm{n}=1001)$, e uma menor proporção de atendimentos individuais com PEM (28,4\%, n=293).

$\mathrm{Na} \mathrm{AB}$, as ofertas terapêuticas foram, predominantemente, a consulta médica $(70,1 \%, \mathrm{n}=1947)$ e o atendimento individual com PEM (66,6\%, $\mathrm{n}=1851)$. Contudo, pontuam-se outras atividades que, apesar de baixa proporção, diferem do funcionamento dos demais pontos de atenção, como as atividades comunitárias $(10,3 \%, \mathrm{n}=287)$ e as visitas domiciliares $(8,4 \%, \mathrm{n}=233)$.
Destaca-se que, além da $\mathrm{AB}$, todos os serviços apresentaram baixa proporção de prestação de cuidados clínicos, havendo destaque apenas para os tratamentos clínicos ou encaminhamentos para esse fim no Caps-II (17,4\%, n=72) e a aferição dos sinais vitais no Caps-AD (39,3\%, n=141). Entre os usuários do CAPS-II e do Ambulatório, a aferição dos sinais vitais foi realizada apenas em $7,3 \%(n=30)$ e $4,2 \%(n=43)$ dos usuários, respectivamente. Informações relativas ao registro de comorbidades, tabagismo, etilismo e uso de outras SPA entre os usuários podem ser observadas na tabela 3 .

Tabela 3. Registro de comorbidades, tabagismo, etilismo e uso de outras SPA entre usuários incluídos no estudo de acordo com o ponto de atenção a que estão vinculados ( $n=4582)$

\begin{tabular}{|c|c|c|c|c|c|c|c|c|}
\hline & \multicolumn{2}{|c|}{ Caps-II } & \multicolumn{2}{|c|}{ Caps-AD } & \multicolumn{2}{|c|}{ Ambulatório } & \multicolumn{2}{|c|}{ Atenção Básica } \\
\hline & $\mathrm{n}$ & $\%$ & $n$ & $\%$ & $\mathrm{n}$ & $\%$ & $\mathbf{n}$ & $\%$ \\
\hline \multicolumn{9}{|l|}{ Hipertensão } \\
\hline Sim & 73 & 17,7 & 17 & 4,7 & 60 & 5,8 & 847 & 31,5 \\
\hline Não & 175 & 42,4 & 100 & 27,8 & 23 & 2,2 & 337 & 12,1 \\
\hline Sem registro & 165 & 39,9 & 242 & 67,4 & 949 & 92,0 & 1567 & 56,4 \\
\hline \multicolumn{9}{|l|}{ Diabetes } \\
\hline Sim & 51 & 12,3 & 7 & 1,9 & 28 & 2,7 & 337 & 12,1 \\
\hline Não & 187 & 45,3 & 104 & 29,0 & 32 & 3,1 & 493 & 17,8 \\
\hline Sem registro & 175 & 42,4 & 248 & 69,1 & 972 & 94,2 & 1948 & 70,1 \\
\hline \multicolumn{9}{|l|}{ Tabagismo } \\
\hline Sim & 103 & 24,9 & 63 & 17,5 & 11 & 1,1 & 185 & 6,7 \\
\hline Não & 228 & 55,2 & 41 & 11,4 & 23 & 2,2 & 210 & 7,6 \\
\hline Sem registro & 82 & 19,9 & 255 & 71,1 & 998 & 96,7 & 2383 & 85,8 \\
\hline \multicolumn{9}{|l|}{ Etilismo } \\
\hline Sim & 54 & 13,1 & 219 & 61,0 & 5 & 0,5 & 107 & 3,9 \\
\hline Não & 271 & 65,6 & 15 & 4,2 & 21 & 2,0 & 176 & 6,3 \\
\hline Sem registro & 88 & 21,3 & 125 & 34,8 & 1006 & 97,5 & 2495 & 89,8 \\
\hline \multicolumn{9}{|c|}{ Uso de outras SPA } \\
\hline Sim & 34 & 8,2 & 202 & 56,3 & 5 & 0,5 & 56 & 2,0 \\
\hline Não & 278 & 67,3 & 25 & 6,9 & 1 & 0,1 & 74 & 2,7 \\
\hline Sem registro & 101 & 24,5 & 132 & 36,8 & 1026 & 99,4 & 2648 & 95,3 \\
\hline
\end{tabular}

Fonte: elaboração própria.

SPA= Substâncias Psicoativas. 
A falta de informações referentes à comorbidade de hipertensão e diabetes impossibilitou a identificação de sua ocorrência, especialmente no Caps-AD, no ambulatório e na $\mathrm{AB}$, locais onde mais da metade dos prontuários não disponibilizava essas informações. Já no Caps-II, por mais que a proporção de usuários sem essas informações seja alta, foi possível observar que $17,7 \%(\mathrm{n}=73)$ dos usuários eram apontados como hipertensos e $12,3 \%(n=51)$ como diabéticos.

Quanto ao tabagismo, apesar da falta de informações em 19,9\% (n=82) dos casos, foi possível observar no Caps-II uma proporção de 24,9\% (n=103) de usuários fumantes. Já com relação ao etilismo, no Caps-AD, embora não houvesse informações acerca de 34,8\% dos usuários, $61 \%(n=219)$ deles foram identificados como etilistas.

Com relação ao uso de SPA, destaca-se, também, a ausência de informações em parte considerável dos usuários do Caps-II e do Caps-AD, contudo, foi possível identificar uma proporção de uso de $8,2(n=34)$ e $56,3 \%$ $(n=202)$, respectivamente. Entre usuários do ambulatório e da $\mathrm{AB}$, a falta de informações foi de 99,4\% (n=1026) e 95,3\% ( $n=2648)$, respectivamente.

\section{Discussão}

Embora se apoie em dados descritivos, este estudo permite a comparação do desempenho de diferentes modalidades de atendimento em saúde mental em um mesmo cenário. Mesmo que as populações assistidas pelos serviços tenham características distintas, os aspectos investigados dizem respeito a informações e cuidados básicos que deveriam ser transversais para a população estudada. Nesse sentido, os dados apresentados sugerem uma heterogeneidade importante no processo de trabalho dos serviços e destacam alguns elementos que precisam ser problematizados.

De forma geral, foi evidenciada uma grande proporção de falta de registros acerca de informações relevantes sobre os usuários. No campo social, destacam-se aspectos como nível de escolaridade e situação laboral. Essa situação, remonta à ideia acerca da existência de variáveis fortes e variáveis sombra na constituição dos elementos que assumem papel de destaque na avaliação da evolução de um transtorno mental e na estratégia de intervenção adotada ${ }^{13}$. As variáveis fortes estariam relacionadas ao diagnóstico, à gravidade do quadro e ao histórico da doença, enquanto as variáveis sombra se relacionam com os recursos individuais da pessoa com transtorno mental, como capacidade intelectual, grau de informação etc. Argumenta-se, ainda, que essas últimas variáveis seriam deixadas à sombra por serem irrelevantes no que diz respeito à evolução da enfermidade e à estratégia de intervenção, entretanto, é provável que um usuário piore muito mais pela falta desses recursos do que pelo tipo de diagnóstico, por exemplo.

Destaca-se a falta de informações referentes a escolaridade e situação laboral entre os usuários do Caps-AD. A falta dessas informações, assim como proposto por outros autores ${ }^{13}$, pode prejudicar a clínica. A proporção de inserção escolar, por exemplo, já foi reconhecida por trabalhos prévios ${ }^{\mathbf{1 4}}$ como um indicador de qualidade em serviços de saúde mental. Quanto ao ambulatório e à $\mathrm{AB}$, cujos casos são majoritariamente relacionados aos transtornos neuróticos e afetivos, cabe reconhecer que esses transtornos estão entre as principais causas de perda de dias de trabalho, sendo os transtornos ansiosos a segunda maior causa dos afastamentos laborais ${ }^{15}$. Além disso, de forma geral, é preciso pontuar a impossibilidade de acessar aspectos como raça/cor e sexualidade dos usuários. Aspectos que, no campo da saúde mental, são marcadores importantes das experiências vivenciadas pelos usuários, tanto no acometimento dos transtornos mentais como no percurso para o cuidado e a assistência recebida.

Como contraponto, cabe, ainda, ressaltar que, além da priorização de registro das questões relativas à doença e sua evolução, outro 
fator que contribui para pobreza de informações acerca dos usuários de serviços de saúde mental no País como um todo é a falta de padronização das variáveis para registro nos prontuários. Nesse sentido, experiências prévias $^{\mathbf{1 4}}$ demonstram que os serviços poderiam se beneficiar da elaboração de instrumentos que norteiem tanto a coleta e o registro dos dados como a sua atualização periódica.

Foram evidenciadas diferenças importantes entre os serviços e a forma de acesso dos usuários a eles, apontando, assim, para formas diferentes de relacionamento com a rede de atenção. Destaca-se, por exemplo, a alta proporção de usuários que acessaram o ambulatório e o Caps-AD por demanda espontânea. Embora seja um movimento esperado para o Caps-AD, dada a persistente dificuldade de outros serviços, em especial, a $\mathrm{AB}$, em lidar com o usuário de SPA ${ }^{16,17}$, a proporção de usuários do ambulatório que acessaram o serviço de forma direta sugere que este tem se configurado como uma das principais portas de entrada para os cuidados em saúde mental na rede estudada. Vale ressaltar, ainda, que, além de configurar uma porta de entrada, ele pode estar assumindo uma posição de gatekeeper com relação ao Caps-II, uma vez que foi o serviço responsável pela maioria dos encaminhamentos de usuários que acessaram o Caps-II via outro serviço especializado.

Com relação ao Caps-AD, pontua-se, ainda, a proporção significativa de usuários que acessaram o serviço através do encaminhamento de serviços privados, amplamente representados pelas CTs. Embora não sejam equipamentos novos na conjuntura de cuidados em saúde mental, a validação desses serviços, enquanto serviços de saúde, proposta pela portaria $\mathrm{n}^{\circ}$ 3.588 , de 21 de dezembro de $2017^{6}$, inclusive com financiamento público, necessita ser urgentemente discutida junto à sociedade civil. Ressalta-se que o encaminhamento para os serviços do sistema público de saúde, em sua maioria, não representa um movimento de integração, mas, sim, o uso do sistema público para suprir a falta de equipe técnica das CTs, bem como uma divisão oportunista da clientela, na medida em que usuários que tendem a apresentar respostas limitadas são encaminhados aos Caps-AD ${ }^{7}$.

A falta de atribuição de diagnóstico entre os usuários do Caps-AD e da $\mathrm{AB}$ chama atenção. Enquanto no Caps-AD a proporção de usuários que está no serviço há menos de um ano, o baixo número de atendimentos recebidos pela maioria dos usuários e a baixa proporção de usuários que passaram em consulta médica poderiam explicar parte desse cenário, existem na literatura subsídios que levam a crer que, na $A B$, essa realidade esteja associada a uma dificuldade dos profissionais em atribuir diagnósticos de transtornos mentais ${ }^{18,19}$.

Ainda quanto aos diagnósticos, foi possível evidenciar que a demanda de cuidados no Ambulatório foi semelhante à demanda observada nos serviços de AB estudados. Em ambos os serviços, a maioria dos casos assistidos correspondiam aos diagnósticos de transtornos neuróticos e transtornos afetivos unipolares. Especialmente para AB, esses dados corroboram achados de estudos prévios $^{20}$. Contudo, cabe destacar que, neste estudo, foram incluídas apenas as unidades de AB que operavam no modelo de ESF (13 unidades), havendo, ainda, 6 unidades que operam no modelo tradicional. Esse dado é relevante na medida em que pode explicar, em parte, a semelhança entre a demanda do ambulatório e da AB. Sugere-se como hipótese que parte dos casos atendidos no ambulatório corresponde aos casos não absorvidos pelas unidades tradicionais de $\mathrm{AB}$, que, em contraponto às unidades ESF, não contam com apoio matricial, sendo, assim, menos propensas a assumir a coordenação do cuidado de usuários com queixas de saúde mental.

Os serviços estudados apresentaram diferenças significativas quanto às ofertas terapêuticas realizadas. No Caps-II, foi observada uma alta prevalência na oferta de consultas médicas em saúde mental e realização de atendimentos individuais por profissionais não médicos. Por outro lado, a oferta de atendimentos coletivos 
e de oficinas terapêuticas foi baixa, estando, assim, em desacordo com as expectativas para o processo de trabalho dos Caps. Destaca-se que, no contexto de reinserção social e abordagem biopsicossocial, as atividades grupais e oficinas deveriam constituir uma das principais formas de tratamento oferecidas nesses serviços ${ }^{21}$, ademais, sua utilização tem sido associada à melhora da função social de pessoas com transtornos psíquicos, tanto no cotidiano familiar como na comunidade 22 .

No Caps-AD, por outro lado, observou-se uma alta prevalência de atendimentos coletivos, embora a oferta de oficinas terapêuticas também tenha sido baixa. Destaca-se, no entanto, a baixa proporção de usuários que receberam consultas médicas de saúde mental no ano que antecedeu a coleta. Trata-se de um aspecto que pode estar atrelado tanto ao funcionamento do serviço em questão, que valorizaria o atendimento coletivo em detrimento do individual, representando, assim, um problema para questões que poderiam ser mais bem exploradas individualmente, como um aspecto relacionado à incompatibilidade da capacidade de pessoal médico à demanda do serviço. Para este segundo caso, são recorrentes na literatura os relatos de que, frente à escassez de profissionais, as filas de atendimento médico individual acabam se acumulando ${ }^{23,24}$.

Deve-se salientar que foi observada uma baixa utilização de recursos comunitários, especialmente as visitas domiciliares, chamando atenção para um movimento de engessamento dos serviços e priorização das práticas intramuros. Esse movimento também foi observado no estudo de Onocko-Campos et al. ${ }^{25}$ que avaliou a atuação dos Caps em quatro centros urbanos do Brasil. No estudo em questão, os autores evidenciaram que a prevalência de atividades comunitárias variou de $4 \%$ a $11 \%$, enquanto a proporção de visitas domiciliares oscilou entre $4 \%$ e $9 \%$. Somada à predominância de um baixo número de atendimentos recebidos pelos usuários no último ano, essa perspectiva chama atenção, ainda, para uma possível ambulatorização desses serviços.

Exceto pelo ambulatório, foi possível observar que, assim como evidenciado por outros estudos ${ }^{25}$, o atendimento com PEM tem se configurado como uma importante ação do cuidado em saúde mental ao redor do País. Esse dado reforça a necessidade de avançar nos esforços para inclusão dos diversos integrantes da equipe multiprofissional em ações de qualificação do cuidado em saúde mental. No ambulatório, por sua vez, houve uma baixa proporção de atendimento individual com PEM, mesmo que essa tenha sido a única modalidade de atendimento oferecida além da consulta médica.

Destaca-se que estudos recentes ${ }^{26}$ têm contextualizado o papel dos ambulatórios na rede de saúde mental e apontam para eles como local onde um trabalho clínico e psicoterápico, não intensivo, poderia ser feito com uma clientela por um determinado período. Dessa forma, usuários que não atendem aos critérios de inclusão nos Caps, mas que necessitam de cuidados especializados em saúde mental que não são encontrados na $\mathrm{AB}$, poderiam ser beneficiados. Contudo, é importante observar que, para isso, os ambulatórios precisam estar alinhados com a perspectiva da atenção psicossocial, indo, assim, em oposição à visão organicista oferecida pelo modelo biomédico, amplamente reproduzida nesses serviços ${ }^{27}$. No ambulatório estudado, por exemplo, para além da priorização das consultas médicas, destacou-se a baixa prevalência de cuidados clínicos ofertados aos usuários, além do baixo registro acerca de comorbidades ou mesmo de hábitos como tabagismo, etilismo e uso de outras SPA, todos relevantes nesse grupo de pacientes.

A baixa oferta de cuidados clínicos também foi observada no Caps-II e no Caps-AD, especialmente no que se refere à aferição do peso e dos sinais vitais. Trata-se de um achado preocupante na medida em que estudos anteriores conduzidos no País apontaram uma alta prevalência de obesidade entre usuários de Caps $^{\mathbf{2 8}}$. Destaca-se que a obesidade, por 
sua vez, tende a favorecer a ocorrência de comorbidades como a hipertensão e o diabetes, cujas chances de manifestação entre pessoas com transtornos mentais são 2,08 e 3,51 vezes maiores que na população em geral, respectivamente ${ }^{29}$.

Pontua-se, ainda, a baixa prevalência de registro da presença ou não de comorbidades, observada em todos os serviços avaliados. No Caps-AD, no ambulatório e na $\mathrm{AB}$, mais da metade dos usuários não contava com esses registros em seus prontuários. Essa realidade é particularmente alarmante no Caps-AD, que, segundo a portaria que estabelece as modalidades dos Caps no País, deveria contar com um médico clínico responsável pela triagem, avaliação e acompanhamento das intercorrências clínicas de seus usuários ${ }^{30}$. Já no Caps-II, mesmo com uma proporção expressiva de registros faltantes, foi possível observar uma prevalência de diabetes 1,98 vezes maior que a observada para a população geral brasileira ${ }^{31}$, chamando atenção para a necessidade de qualificar o cuidado desses usuários, buscando, especialmente, integrar o cuidado fornecido nos serviços especializados de saúde mental com a $\mathrm{AB}$.

Nesse sentido, destaca-se que a $A B$ foi o ponto de atenção onde os usuários receberam cuidados clínicos em maior proporção, apresentando prevalências superiores a 90\% nos itens: aferição do peso, aferição dos sinais vitais e anamnese e exame físico. Esses achados reforçam a ideia de que a $\mathrm{AB}$ é um ponto de atenção potente e necessário para o cuidado das pessoas com transtornos mentais. Contudo, estudos conduzidos anteriormente no País têm demonstrado que, após acessar os serviços especializados de saúde mental, muitos usuários perdem o contato com as equipes de $\mathrm{AB}^{32}$. Dessa forma, cada vez mais se faz necessário lançar mão de estratégias que visem à integração desses serviços.

Na última década, uma estratégia que tem se destacado é o apoio matricial ${ }^{33}$. Trata-se de um modelo de intervenção pedagógico-terapêutica que visa a favorecer a troca de informações e ampliar a corresponsabilização pelos usuários entre as equipes de diferentes níveis de atenção ${ }^{34}$. Sua ação tem se mostrado relevante na incorporação de novas possibilidades no cuidado em saúde mental, contudo, uma série de desafios ainda está posta, como, por exemplo, a persistência de um entendimento biológico sobre o processo saúde-doença, em detrimento do olhar biopsicossocial, havendo uma forte centralidade do processo de trabalho no modelo curativo e individual ${ }^{33}$. Pontua-se, então, a necessidade de um investimento maciço em formação e capacitação dos profissionais, tanto para o trabalho articulado em rede como para o rastreio e monitoramento das necessidades em saúde dos usuários que convivem com um transtorno mental.

\section{Conclusões}

Foi evidenciada uma alta proporção de informações faltantes acerca de características que são relevantes para o cuidado dos indivíduos estudados, sugerindo, assim, que questões relativas à condição clínica e socioeconômica dos usuários podem estar sendo negligenciadas. Além disso, foram evidenciadas diferenças importantes entre os serviços no que diz respeito ao seu funcionamento, bem como ao diálogo estabelecido com os outros pontos de atenção da rede de saúde.

Além da realização de consultas médicas, a ampla oferta de atendimentos individuais com profissionais da equipe multidisciplinar se mostrou como um ponto forte na maioria dos serviços estudados, reforçando, assim, a necessidade de valorização e engajamento da equipe interdisciplinar no cuidado em saúde mental. Por outro lado, foi observada uma baixa utilização dos recursos coletivos, como as oficinas terapêuticas, evidenciando a necessidade de retomada e reafirmação de práticas já consagradas na perspectiva da reabilitação psicossocial.

A rede estudada se caracterizou, ainda, pela baixa utilização dos recursos comunitários e 
*Orcid (Open Researcher and Contributor ID). das visitas domiciliares, bem como pela baixa proporção de cuidados clínicos oferecidos aos usuários nos serviços especializados de saúde mental. Por outro lado, a AB foi responsável por apresentar altas proporções de cuidados clínicos aos seus usuários, destacando-se, assim, enquanto um serviço potente e necessário para o cuidado das pessoas com transtornos mentais.

Dado o desenho do estudo, salienta-se a limitação de seu caráter descritivo, além da grande proporção de dados faltantes em algumas das variáveis estudadas. Destaca-se, no entanto, que, assim como demonstrado ao longo da discussão, os dados apresentados dialogam com problemas que são transversais ao sistema nacional de saúde, bem como com problemáticas atuais e relevantes que vêm sendo discutidas ao redor do mundo.

\section{Colaboradores}

Treichel CAS (0000-0002-0440-9108)* e Onocko Campos RT (0000-0003-0469-5447)* contribuíram igualmente para a elaboração do manuscrito.

\section{Referências}

1. Global Burden of Disease Collaborative Network. Global Burden of Disease Study 2016 (GBD 2016) Results. Seattle, United States: Institute for Health Metrics and Evaluation (IHME). [acesso em 2020 fev 17]. Disponível em: http://ghdx.healthdata.org/ gbd-results-tool.

2. World Health Organization. Depression and Other Common Mental Disorders: Global Health Estimates. Geneva: WHO; 2017.

3. Brasil. Ministério da Saúde, Secretaria de Atenção à Saúde, Coordenação Geral de Saúde Mental, Álcool e Outras Drogas, 2016. Saúde Mental no SUS: Cuidado em Liberdade, Defesa de Direitos e Rede de Atenção Psicossocial. Relatório de Gestão 2011-2015. [acesso em 2020 fev 17]. Disponível em: https://portalarquivos2.saude.gov.br/images/pdf/2016/junho/27/Relat--rio-Gest--o-2011-2015---.pdf.
4. Brasil. Ministério da Saúde. Portaria n ${ }^{\circ} 3.088$, de 23 de dezembro de 2011. Institui a Rede de Atenção Psicossocial para pessoas com sofrimento ou transtorno mental e com necessidades decorrentes do uso de crack, álcool e outras drogas, no âmbito do Sistema Único de Saúde (SUS). Diário Oficial da União. 26 Dez 2011

5. Onocko-Campos RT. Mental health in Brazil: strides, setbacks, and challenges. Cad. Saúde Pública. 2019; 35(11): e00156119.

6. Brasil. Ministério da Saúde. Portaria n ${ }^{0} 3.588$, de 21 de dezembro de 2017. Altera as Portarias de Consolidação nº 3 e nº 6 , de 28 de setembro de 2017, para dispor sobre a Rede de Atenção Psicossocial, e dá outras providências. Diário Oficial da União. 22 Dez 2017. 
7. Guimarães TAA, Rosa LCS. A remanicomialização do cuidado em saúde mental no Brasil no período de 2010-2019: análise de uma conjuntura antirreformista. Soc. Quest. 2019; 44(2):111-138.

8. World Health Organization. Mental health action plan 2013-2020. Geneva: WHO; 2013.

9. Patel V, Saxena S, Lund C, et al. The Lancet Commission on global mental health and sustainable development. Lancet. 2018; (392):1553-98.

10. Storm M, Fortuna KL, Gill EA, et al. Coordination of services for people with serious mental illness and general medical conditions: Perspectives from rural northeastern United States. Psychiatr Rehabil J. 2020; 43(3):234-243.

11. Reilly S, Olier I, Planner C, et al. Inequalities in physical comorbidity: a longitudinal comparative cohort study of people with severe mental illness in the UK. BMJ Open. 2015; (5):e009010.

12. Conselho Nacional de Saúde. Resolução no 466 , de 12 de dezembro de 2012. Aprova diretrizes e normas regulamentadoras de pesquisas envolvendo seres humanos. Diário Oficial da União. 12 Dez 2012.

13. Saraceno B. Manual de saúde mental. São Paulo: Hucitec; 1994

14. Bustamante V, Onocko-Campos R, Silva AA, et al. Indicadores para avaliação de Centros de Atenção Psicossocial Infantojuvenil (Capsi): resultados de uma pesquisa-intervenção. Interface (Botucatu). 2020; (24):e190276

15. Ribeiro HKP, Santos JDM, Silva MG, et al. Transtornos de ansiedade como causa de afastamentos laborais. Rev. bras. saúde ocup. 2019; (44):el.

16. Paula ML, Jorge MSB, Vasconcelos MGF, et al. Assistência ao usuário de drogas na atenção primária à saúde. Psicol. estud. 2014; 19(2):223-233.

17. Souza FE, Ronzani TM. Desafios às práticas de re- dução de danos na atenção primária à saúde. Psicol. estud. 2018; (23):e2306.

18. Tanaka OY, Ribeiro EL. Ações de saúde mental na atenção básica: caminho para ampliação da integralidade da atenção. Ciênc. Saúde Colet. 2009; 14(2):477486.

19. Campos-Junior A. Estudo sobre práticas de cuidado em saúde mental na atenção primária: o caso de um município do interior do estado do Rio de Janeiro. [dissertação]. Rio de Janeiro: Escola Nacional de Saúde Pública Sergio Arouca, Fundação Oswaldo Cruz; 2013.

20. Gonçalves DA, Mari JJ, Bower P, et al. Brazilian multicentre study of common mental disorders in primary care: rates and related social and demographic factors. Cad. Saúde Pública. 2014; 30(3):623-632.

21. Brasil. Ministério da Saúde. Saúde mental no SUS: os centros de atenção psicossocial. Brasília, DF: Ministério da Saúde. [acesso em 2020 fev 17]. Disponível em http://portal.saude.gov.br/portal/arquivos/pdf/ manual_caps.pdf.

22. Ibiapina ARS, Monteiro CFS, Alencar DC, et al. Oficinas Terapêuticas e as mudanças sociais em portadores de transtorno mental. Escola Anna Nery. 2017; 21(3):e20160375.

23. Lacerda CB, Fuentes-Rojas M. Significados e sentidos atribuídos ao Centro de Atenção Psicossocial Álcool e outras Drogas (CAPS AD) por seus usuários: um estudo de caso. Interface (Botucatu). 2017; 21(61)363372.

24. Silva SN, Lima MG. Avaliação da estrutura dos Centros de Atenção Psicossocial da região do Médio Paraopeba, Minas Gerais. Epidemiol. Serv. Saúde. 2017; 26(1):149-160.

25. Onocko-Campos RT, Amaral CEM, Saraceno B, et al. Atuação dos Centros de Atenção Psicossocial em quatro centros urbanos no Brasil. Rev. Panam. Salud Publica. 2018; (42):el13. 
26. Damous I, Erlich H. O ambulatório de saúde mental na rede de atenção psicossocial: reflexões sobre a clínica e a expansão das políticas de atenção primária. Physis. 2017; 27(4):911-932.

27. Brandão-Junior PMC, Canavêz F, Ramos PL. Entre saúde e educação: sobre um ambulatório de saúde mental infantojuvenil. Interface (Botucatu). 2017; 21(62):699-709.

28. Jardim VMR, Treichel CAS, Kantorski LP, et al. Overweight and obesity among individuals with mental disorders in southern Brazil. J Nurs Health. 2017; 7(3):e177301.

29. Woodhead C, Ashworth M, Schofield P, et al. Patterns of physical co-/multi-morbidity among patients with serious mental illness: a London borough-based cross-sectional study. BMC fam pract. 2014; 15(117):1-9.

30. Brasil. Ministério da Saúde. Portaria n ${ }^{\circ} 336$, de 19 de fevereiro de 2002. Estabelece as modalidades dos serviços CAPS no âmbito do Sistema Único de Saúde (SUS). Diário Oficial da União. 20 Fev 2002.

31. Malta DC, Duncan BB, Schmidt MI, et al. Prevalência de diabetes mellitus determinada pela hemoglo- bina glicada na população adulta brasileira, Pesquisa Nacional de Saúde. Rev. bras. epidemiol. 2019; 22(supl2):e190006.

32. Amaral CE, Onocko-Campos R, Oliveira PRS, et al. Systematic Review of Pathways to Mental Health Care in Brazil: Narrative Synthesis of Quantitative and Qualitative Studies. Int J Ment Health Syst. 2018; (12):65.

33. Treichel CAS, Campos RTO, Campos GWS. Impasses e desafios para consolidação e efetividade do apoio matricial em saúde mental no Brasil. Interface (Botucatu). 2019; (23):e180617.

34. Campos GWS, Domitti AC. Apoio matricial e equipe de referência: uma metodologia para gestão do trabalho interdisciplinar em saúde. Cad. Saúde Pública. 2007; 23(2):399-407.

Recebido em 08/06/2021

Aprovado em 12/10/202

Conflito de interesses: inexistente

Suporte financeiro: a pesquisa que originou este estudo foi financiada pela Fundação de Amparo à Pesquisa do Estado de São Paulo (Fapesp), processo no 2018/10366-6. O presente trabalho foi realizado com apoio da Coordenação de Aperfeiçoamento de Pessoal de Nível Superior - Brasil (Capes) -, Código de Financiamento 001 